\title{
EVOLUTION OF THE RADIO REMNANT OF SUPERNOVA 1987A: MORPHOLOGICAL CHANGES FROM DAY 7000
}

\author{
C.-Y. NG $^{1}$, G. Zanardo ${ }^{2}$, T. M. Potter ${ }^{2}$, L. Staveley-Smith ${ }^{2,3}$, B. M. Gaensler ${ }^{3,4}$, R. N. Manchester ${ }^{5}$, And A. K. Tzioumis ${ }^{5}$ \\ ${ }^{1}$ Department of Physics, The University of Hong Kong, Pokfulam Road, Hong Kong; ncy @ bohr.physics.hku.hk \\ ${ }^{2}$ International Centre for Radio Astronomy Research (ICRAR), The University of Western Australia, Crawley, WA 6009, Australia \\ ${ }^{3}$ Australian Research Council, Centre of Excellence for All-sky Astrophysics (CAASTRO) \\ ${ }^{4}$ Sydney Institute for Astronomy, School of Physics, The University of Sydney, NSW 2006, Australia \\ ${ }^{5}$ CSIRO Astronomy and Space Science, Australia Telescope National Facility, Marsfield, NSW 1710, Australia \\ Received 2013 August 19; accepted 2013 September 10; published 2013 October 22
}

\begin{abstract}
We present radio imaging observations of supernova remnant 1987A at $9 \mathrm{GHz}$, taken with the Australia Telescope Compact Array over 21 years from 1992 to 2013. By employing a Fourier modeling technique to fit the visibility data, we show that the remnant structure has evolved significantly since day 7000 (mid-2006): the emission latitude has gradually decreased such that the overall geometry has become more similar to a ring structure. Around the same time, we find a decreasing trend in the east-west asymmetry of the surface emissivity. These results could reflect the increasing interaction of the forward shock with material around the circumstellar ring, and the relative weakening of the interaction with the lower-density material at higher latitudes. The morphological evolution caused an apparent break in the remnant expansion measured with a torus model, from a velocity of $4600_{-200}^{+150} \mathrm{~km} \mathrm{~s}^{-1}$ between day 4000 and 7000 to $2400_{-200}^{+100} \mathrm{~km} \mathrm{~s}^{-1}$ after day 7000 . However, we emphasize that there is no conclusive evidence for a physical slowing of the shock at any given latitude in the expanding remnant, and that a change of radio morphology alone appears to dominate the evolution. This is supported by our ring-only fits which show a constant expansion of $3890 \pm 50 \mathrm{~km} \mathrm{~s}^{-1}$ without deceleration between days 4000 and 9000 . We suggest that once the emission latitude no longer decreases, the expansion velocity obtained from the torus model should return to the same value as that measured with the ring model.
\end{abstract}

Key words: circumstellar matter - ISM: supernova remnants - radio continuum: ISM - shock waves supernovae: individual (SN 1987A)

Online-only material: color figures

\section{INTRODUCTION}

The remarkable supernova (SN) 1987A in the Large Magellanic Cloud has enabled detailed studies of many fields in astrophysics from massive star evolution to the SN explosion mechanism to the earliest stage of supernova remnants (SNRs; see reviews in Immler et al. 2007). As well as being the brightest SN over the past $400 \mathrm{yr}$, SN 1987A was a highly unusual event. In particular, the progenitor was surrounded by a peculiar triple-ring nebula (Burrows et al. 1995), which could have resulted from a binary merger of the progenitor $20,000 \mathrm{yr}$ prior to the explosion (Morris \& Podsiadlowski 2007). Over the past decade, the remnant has undergone a major evolution since the shock collision with the inner equatorial ring, resulting in rapid brightening of the radio and soft X-ray emissions (see Zanardo et al. 2010; Helder et al. 2013 and references therein).

Radio emission of SNR 1987A is believed to be non-thermal synchrotron radiation emitted by energetic particles accelerated in shocks. Since the remnant emerged in mid-1990 (Turtle et al. 1990; Staveley-Smith et al. 1992), it has been monitored regularly at different frequencies using the Australia Telescope Compact Array (ATCA; see Staveley-Smith et al. 2007; Zanardo et al. 2010 and references therein). The flux density was found to have increased exponentially from day 5000 to 8000 with a progressively flatter spectrum (Zanardo et al. 2010), indicating increasingly efficient particle acceleration processes.

ATCA imaging observations at $9 \mathrm{GHz}$ have been taken about twice a year since 1992 (Gaensler et al. 1997; Manchester et al. 2002; Ng et al. 2008, hereafter N08). With the source flux increase and various upgrades to the telescope, the remnant has been resolved at progressively higher frequencies, from $18 \mathrm{GHz}$ to $36 \mathrm{GHz}$ to $44 \mathrm{GHz}$ to $94 \mathrm{GHz}$ (Manchester et al. 2005; Potter et al. 2009; Zanardo et al. 2013; Lakićević et al. 2012), and also with very long baseline interferometry (VLBI) at $1.4 \mathrm{GHz}$ and $1.7 \mathrm{GHz}$ (Tingay et al. 2009; Ng et al. 2011a). The remnant shows similar structure at all these frequencies, and can be described as a thin shell with an asymmetric surface brightness distribution along the east-west direction. A thinshell model was used to quantify the remnant structure in early studies (Staveley-Smith et al. 1993b; Gaensler et al. 1997). N08 developed a three-dimensional (3D) torus model that can capture the latitude extent of the emission and the east-west asymmetry. Fitting the torus model to observations between 1992 and 2008, a linear expansion of $\sim 4000 \mathrm{~km} \mathrm{~s}^{-1}$ was found up to day 8000 , which is in contrast to the deceleration of the X-ray remnant observed around day 6000 (Racusin et al. 2009; Helder et al. 2013).

In this paper, we report on the latest evolution of the radio morphology of SNR 1987A up to day 9568 after the SN explosion, using $9 \mathrm{GHz}$ ATCA imaging observations taken from 1992 January to 2013 May. The observations and Fourier modeling scheme are described in Sections 2 and 3, respectively, the modeling results are presented in Section 4, and we infer the remnant expansion rate in Section 5. The physical implications of the results are discussed in Section 6.

\section{OBSERVATIONS AND DATA REDUCTION}

Radio imaging of SNR 1987A at $9 \mathrm{GHz}$ have been carried out for $21 \mathrm{yr}$ since $1992 \mathrm{using}$ ATCA in the $6 \mathrm{~km}$ array configuration, 
Table 1

Observational Parameters for the Data Sets Used in This Study

\begin{tabular}{|c|c|c|c|c|c|}
\hline Observing Date & $\begin{array}{l}\text { Days since } \\
\text { Supernova }\end{array}$ & $\begin{array}{c}\text { Array } \\
\text { Configuration }\end{array}$ & $\begin{array}{c}\text { Center Frequency }{ }^{\mathrm{a}} \\
(\mathrm{MHz})\end{array}$ & $\begin{array}{l}\text { Time on } \\
\text { Source (hr) }\end{array}$ & $\begin{array}{l}\text { Epoch Shown } \\
\text { in Figure } 1^{\mathrm{b}}\end{array}$ \\
\hline 1992 Jan 14 & 1786 & $6 \mathrm{~B}$ & 8640 & 12 & $\ldots$ \\
\hline 1992 Mar 20 & 1852 & $6 \mathrm{~A}$ & 8640 & 10 & $\ldots$ \\
\hline 1992 Oct 21 & 2067 & $6 \mathrm{C}$ & 8640,8900 & 13 & 1992.9 \\
\hline 1993 Jan 4 & 2142 & $6 \mathrm{~A}$ & 8640,8900 & 9 & 1992.9 \\
\hline 1993 Jan 5 & 2143 & $6 \mathrm{~A}$ & 8640,8900 & 6 & 1992.9 \\
\hline 1993 Jun 24 & 2313 & $6 \mathrm{C}$ & 8640,8900 & 8 & 1993.6 \\
\hline 1993 Jul 1 & 2320 & $6 \mathrm{C}$ & 8640,8900 & 10 & 1993.6 \\
\hline 1993 Oct 15 & 2426 & $6 \mathrm{~A}$ & 8640,9024 & 17 & 1993.6 \\
\hline 1994 Feb 16 & 2550 & $6 \mathrm{~B}$ & 8640,9024 & 9 & 1994.4 \\
\hline 1994 Jun $27-28$ & 2681 & $6 \mathrm{C}$ & 8640,9024 & 21 & 1994.4 \\
\hline 1994 Jul 1 & 2685 & $6 \mathrm{~A}$ & 8640,9024 & 10 & 1994.4 \\
\hline 1995 Jul 24 & 3073 & $6 \mathrm{C}$ & 8640,9024 & 12 & 1995.7 \\
\hline 1995 Aug 29 & 3109 & $6 \mathrm{D}$ & 8896,9152 & 7 & 1995.7 \\
\hline 1995 Nov 6 & 3178 & $6 \mathrm{~A}$ & 8640,9024 & 9 & 1995.7 \\
\hline 1996 Jul 21 & 3436 & $6 \mathrm{C}$ & 8640,9024 & 14 & 1996.7 \\
\hline 1996 Sep 8 & 3485 & $6 \mathrm{~B}$ & 8640,9024 & 13 & 1996.7 \\
\hline 1996 Oct 5 & 3512 & $6 \mathrm{~A}$ & 8896,9152 & 8 & 1996.7 \\
\hline 1997 Nov 11 & 3914 & $6 \mathrm{C}$ & 8512,8896 & 7 & 1998.0 \\
\hline 1998 Feb 18 & 4013 & $6 \mathrm{~A}$ & 8896,9152 & 10 & 1998.0 \\
\hline 1998 Feb 21 & 4016 & $6 \mathrm{~B}$ & 8512,9024 & 7 & 1998.0 \\
\hline 1998 Sep 13 & 4220 & $6 \mathrm{~A}$ & 8896,9152 & 12 & 1998.9 \\
\hline 1998 Oct 31 & 4268 & $6 \mathrm{D}$ & 8502,9024 & 11 & 1998.9 \\
\hline 1999 Feb 12 & 4372 & $6 \mathrm{C}$ & 8512,8896 & 10 & 1999.7 \\
\hline 1999 Sep 5 & 4577 & $6 \mathrm{D}$ & 8768,9152 & 11 & 1999.7 \\
\hline 1999 Sep 12 & 4584 & $6 \mathrm{~A}$ & 8512,8896 & 14 & 1999.7 \\
\hline 2000 Sep 28 & 4966 & $6 \mathrm{~A}$ & 8512,8896 & 10 & 2000.9 \\
\hline 2000 Nov 12 & 5011 & $6 \mathrm{C}$ & 8512,8896 & 11 & 2000.9 \\
\hline 2001 Nov 23 & 5387 & $6 \mathrm{D}$ & 8768,9152 & 8 & 2001.9 \\
\hline 2002 Nov 19 & 5748 & $6 \mathrm{~A}$ & 8512,8896 & 8 & 2003.0 \\
\hline 2003 Jan 20 & 5810 & $6 \mathrm{~B}$ & 8512,9024 & 9 & 2003.0 \\
\hline 2003 Aug 1 & 6003 & $6 \mathrm{D}$ & 8768,9152 & 10 & 2003.6 \\
\hline 2003 Dec 5 & 6129 & $6 \mathrm{~A}$ & 8512,8896 & 9 & 2004.0 \\
\hline 2004 Jan 15 & 6170 & $6 \mathrm{~A}$ & 8512,8896 & 9 & 2004.0 \\
\hline 2004 May 7 & 6283 & $6 \mathrm{C}$ & 8512,8896 & 9 & 2004.4 \\
\hline 2005 Mar 25 & 6605 & $6 \mathrm{~A}$ & 8512,8896 & 9 & 2005.2 \\
\hline 2005 Jun 21 & 6693 & $6 \mathrm{~B}$ & 8512,8896 & 9 & 2005.5 \\
\hline 2006 Mar 28 & 6973 & $6 \mathrm{C}$ & 8512,8896 & 9 & 2006.2 \\
\hline 2006 Jul 18 & 7085 & $6 \mathrm{~A}$ & 8512,8896 & 9 & 2006.5 \\
\hline 2006 Dec 8 & 7228 & $6 \mathrm{~B}$ & 8512,9024 & 8 & 2006.9 \\
\hline 2008 Jan 4 & 7620 & $6 \mathrm{~A}$ & 8512,9024 & 11 & 2008.0 \\
\hline 2008 Apr 23 & 7730 & $6 \mathrm{~A}$ & 8512,8896 & 11 & 2008.3 \\
\hline 2008 Oct 11 & 7901 & $6 \mathrm{~A}$ & 8512,8896 & 11 & 2008.8 \\
\hline 2009 Jun 6 & 8139 & $6 \mathrm{~A}$ & 9000 & 11 & 2009.4 \\
\hline 2010 Jan 23 & 8370 & $6 \mathrm{~A}$ & 9000 & 11 & 2010.1 \\
\hline 2010 Apr 11 & 8448 & $6 \mathrm{~A}$ & 9000 & 11 & 2010.3 \\
\hline 2011 Jan 25 & 8737 & $6 \mathrm{~A}$ & 9000 & 11 & 2011.1 \\
\hline 2011 Apr 22 & 8824 & $6 \mathrm{~A}$ & 9000 & 11 & 2011.3 \\
\hline 2012 Jan 12 & 9089 & $6 \mathrm{~A}$ & 9000 & 11 & 2012.0 \\
\hline 2012 Jun 5 & 9233 & $6 \mathrm{D}$ & 9000 & 11 & 2012.4 \\
\hline 2012 Sep 1 & 9321 & $6 \mathrm{~A}$ & 9000 & 10 & 2012.7 \\
\hline 2013 Mar 7 & 9509 & $6 \mathrm{~A}$ & 9000 & 11 & 2013.2 \\
\hline 2013 May 5 & 9568 & $6 \mathrm{C}$ & 9000 & 11 & 2013.3 \\
\hline
\end{tabular}

Notes.

${ }^{a}$ Since the $\mathrm{CABB}$ upgrade in mid-2009, data have been recorded over a $2 \mathrm{GHz}$ bandwidth. However, in this analysis we used two $104 \mathrm{MHz}$ sub-bands with center frequencies of $8.512 \mathrm{GHz}$ and $8.896 \mathrm{GHz}$, for a consistency with the bandwidth of pre-CABB data.

${ }^{\mathrm{b}}$ Some data sets have been averaged together to generate the corresponding images in Figure 1 for the listed epoch.

with a typical on-source time of $\sim 10 \mathrm{hr}$ for each observation. In this paper, we analyze the remnant evolution in light of datasets recorded to date, which include recent observations taken since the Compact Array Broadband Backend (CABB; Wilson et al. 2011) upgrade in mid-2009. Table 1 lists the observation parameters. Pre-CABB observations were made in two frequencies with a usable bandwidth of $104 \mathrm{MHz}$ each. While the bandwidth has been greatly increased to $2 \mathrm{GHz}$ since the $\mathrm{CABB}$ upgrade, we restricted our analysis of the CABB data in two sub-bands only, with center frequencies of $8.512 \mathrm{GHz}$ and 


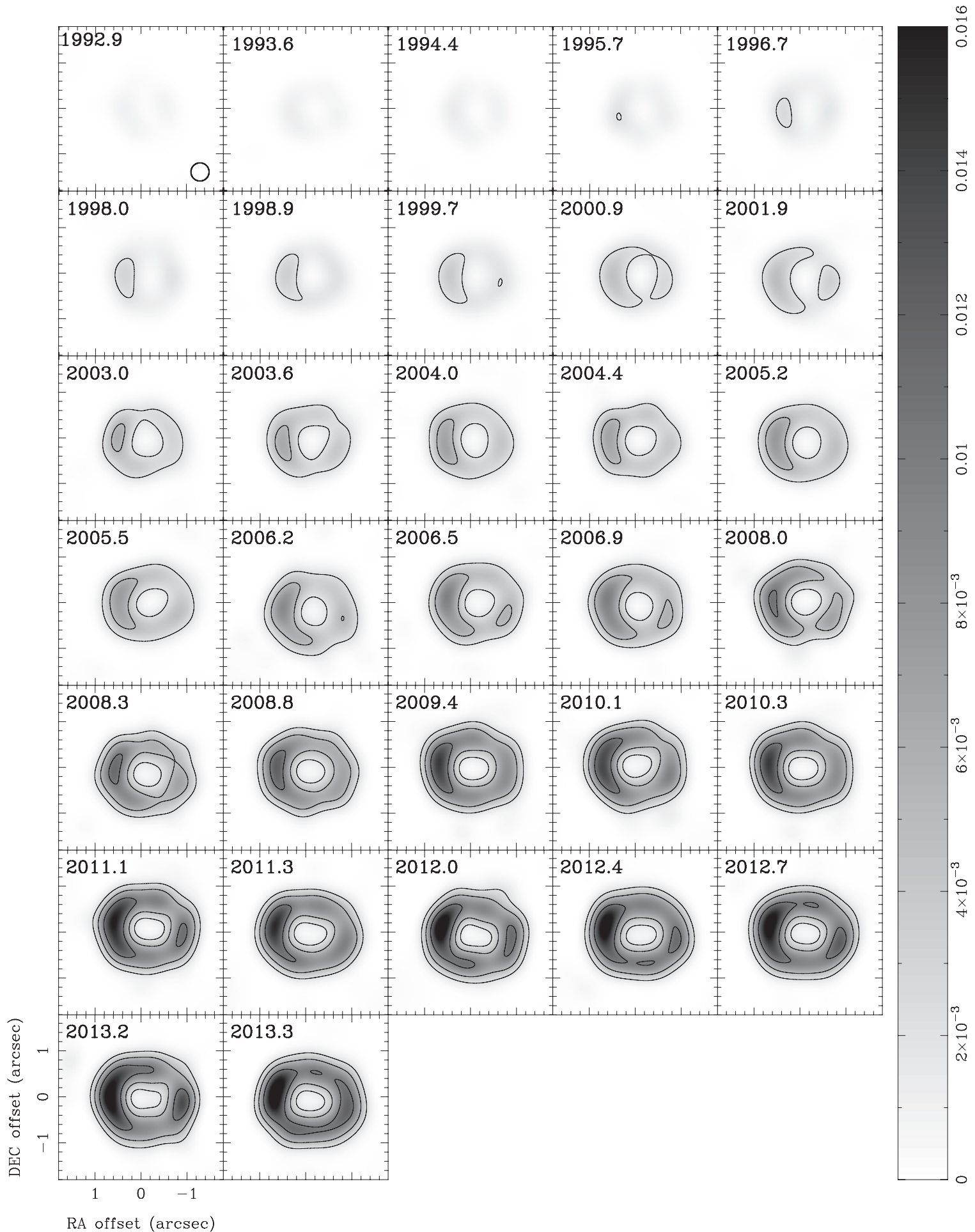

Figure 1. Super-resolved 9 GHz ATCA images of SN 1987A over the period 1992-2013. Some early epochs have been averaged to boost the signal-to-noise ratio (see Table 1). The gray scale is linear ranging from 0 to $16 \mathrm{mJy}_{\text {beam }}^{-1}$ and the contours are at levels of $2,5,10$, and $15 \mathrm{mJy}^{-1}$ beam ${ }^{-1}$. The synthesized beam, which has an FWHM of $00^{\prime \prime} 4$, is shown in the first panel. The typical rms noise level is $0.05 \mathrm{mJy} \mathrm{beam}^{-1}$.

$8.896 \mathrm{GHz}$ and a bandwidth of $104 \mathrm{MHz}$ each, for a consistent comparison with previous data. Note that the data set taken on 2012 December 9 was affected by a storm, leaving only $6 \mathrm{hr}$ of on-source time. It was not used in this study because the $u-v$ sampling is inadequate for high-fidelity imaging, resulting in large rms noise in the final maps $\left(0.5 \mathrm{mJy}^{\mathrm{beam}}{ }^{-1}\right)$.

All data were reduced using the MIRIAD package (Sault et al. 1995). After standard flagging and calibration, we employed self-calibration on data taken after 1996, when the source was detected with a high signal-to-noise ratio (see Gaensler et al. 1997; N08). The visibility data were averaged with fiveminute intervals. The intensity maps formed from the visibility were deconvolved using a maximum entropy algorithm (Gull \& Daniell 1978). We applied a super-resolution technique (Staveley-Smith et al. 1993a) by restoring the cleaned maps with a super-resolved circular beam of FWHM 0"4 (see N08 for the details of the reduction procedure). The final maps have a typical rms noise of $0.05 \mathrm{mJy}$ beam $^{-1}$. Figure 1 shows the 

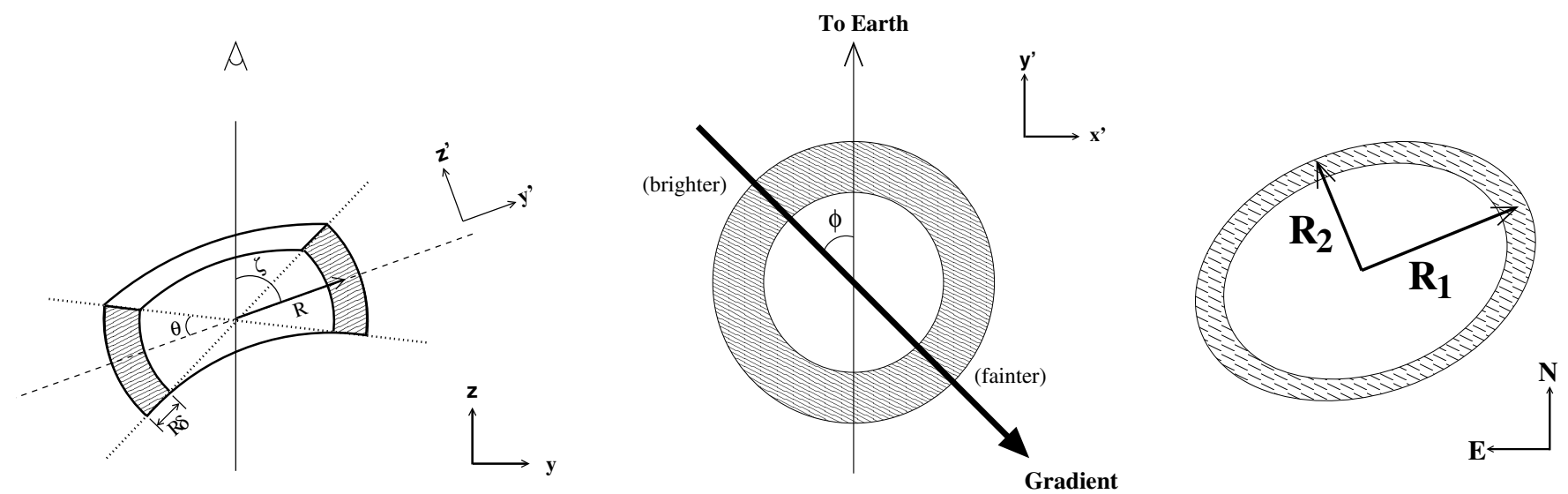

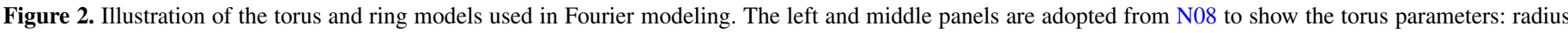

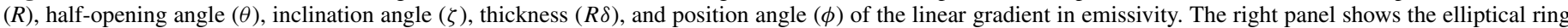

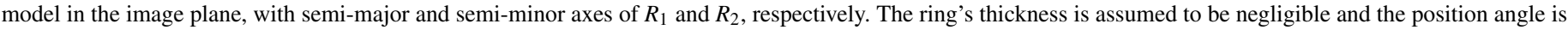
fixed during the fit.

resulting images. The remnant morphology can be described by a circular shell with two bright lobes. The eastern lobe is always brighter than the western lobe. Over the epochs, the remnant has brightened significantly and exhibited a clear expansion.

\section{FOURIER MODELING}

We followed previous studies (e.g., Staveley-Smith et al. 1993b; Gaensler et al. 1997; N08) to assess the remnant geometry in the $u-v$ plane with the Fourier modeling technique. This can give more robust measurements than directly fitting a model in the image plane, since the Fourier domain is where the visibility data are taken and the measurement errors are uncorrelated. We employed two models in this study: a 3D torus as developed by N08 and a two-dimensional (2D) thin elliptical ring.

The torus model is illustrated in Figure 2. It is a truncated shell with eight fitting parameters: flux density, center position (right ascension and declination), radius, half-opening angle $(\theta)$, thickness (as a fraction of the radius), slope (in \%), and direction $(\phi)$ of a linear gradient in the surface emissivity. The actual fitting was performed with a modified version of the MIRIAD task UVFIT (see N08 for details). As an update to the modeling, we employed the $9 \mathrm{GHz}$ light curve reported by Zanardo et al. (2010) to account for the time-of-flight effect that causes the near side of the remnant to appear brighter than the far side. This gives slightly different results compared to N08, however, we emphasize that the differences are minimal and within the uncertainties.

Although N08 obtained confidence intervals of the fitting parameters using a simple bootstrapping technique, we note that the bootstrap samples may not be representative of the true population, and other techniques, such as subsample bootstrapping, are needed (see Kemball \& Martinsek 2005). To avoid complications, we determined the confidence intervals from the covariance matrices. There are a few cases for which this method fails to give sensible results because the best-fit parameters are near the boundaries. We then estimated the confidence intervals by plotting out the $\chi^{2}$ distribution.

In addition to the torus model, an elliptical ring model was also employed to compare with that used in X-ray studies (Racusin et al. 2009; Helder et al. 2013). As in the torus model, we included a linear gradient in the surface emissivity to account for the observed east-west asymmetry. The ring model has seven fitting parameters: flux density, center position, semi-major axis $\left(R_{1}\right)$, semi-minor axis $\left(R_{2}\right)$, and degree and direction of the gradient. The model is illustrated in Figure 2. Since the system is known have an inclination of $\sim 45^{\circ}$ to the line of sight (e.g., Sugerman et al. 2005), we first tried fitting with the ring's aspect ratio fixed at $R_{2} / R_{1}=0.7$, but found that it gives slightly worse statistics than the torus fits. We then allowed both $R_{1}$ and $R_{2}$ to vary, which improves the results and gives comparable $\chi^{2}$ values to the torus model. Same as the torus fits, confidence intervals of the best-fit parameters were determined from the covariance matrix.

\section{RESULTS}

The best-fit model parameters are listed in Tables 2 and 3. As an example, we show in Figure 3 images of the best-fit torus and ring models compared with the actual radio map taken on 2012 September 1, and the maps of the residual (i.e., data minus model) visibilities. The models have successfully captured the characteristic structure of the remnant. With one extra fitting parameter, the torus model provides slightly better fits to the data than the ring model. However, we cannot determine whether the difference in statistics is significant since the standard $F$-test does not apply here. As shown in Figure 3, the ring model underpredicts the flux density at the remnant center, but provides a better fit to the northern rim than the torus model.

Time evolution of the best-fit flux density, radius, opening angle, and degree of asymmetry, which is obtained from the slope of the gradient, are plotted in Figure 4. The torus radius increased linearly at the early epochs until a clear break at day $\sim 7000$. After that, the expansion rate was significantly reduced. It is intriguing that around the same time when the break occurred, both $\theta$ and the asymmetry began to decrease. Since day $\sim 7500$ the model flux density increases at a lower rate than the exponential fit given by Zanardo et al. (2010). For the other parameters not shown in the figure, the torus thickness is not well determined from the fits. As reported by $\mathrm{N} 08$, this is likely smaller than the resolution of the $9 \mathrm{GHz}$ observations (see also $\mathrm{Ng}$ et al. 2011a). The direction of the linear gradient, which identifies the asymmetry of the surface brightness, remains mostly constant over the epochs.

For the ring model fit, it is clear from Figure 4(b) that $R_{1}$ is always smaller than the torus radius. When compared to the X-ray radius reported by Helder et al. (2013), $R_{1}$ has a similar 
Table 2

Best-fit Parameters for the Torus Model with Statistical Uncertainties at a 68\% Confidence Level

\begin{tabular}{|c|c|c|c|c|c|c|c|}
\hline Day & $\begin{array}{l}\text { Flux } \\
(\mathrm{mJy})\end{array}$ & $\begin{array}{l}\text { Radius } \\
\left({ }^{\prime \prime}\right)\end{array}$ & $\begin{array}{l}\text { Half-opening } \\
\text { Angle }\left(^{\circ}\right)\end{array}$ & $\begin{array}{c}\text { Thickness } \\
(\%)\end{array}$ & $\begin{array}{c}\text { Asymmetry } \\
(\%)\end{array}$ & $\begin{array}{l}\phi \\
\left({ }^{\circ}\right)\end{array}$ & $\chi_{v}^{2} / \mathrm{dof}^{\mathrm{a}}$ \\
\hline 1786 & $4.2 \pm 0.2$ & $0.60 \pm 0.10$ & $84_{-20}^{+6}$ & $150 \pm 50$ & $70 \pm 30$ & $187 \pm 16$ & $1.8 / 2107$ \\
\hline 1852 & $4.0 \pm 0.3$ & $0.62 \pm 0.05$ & $80 \pm 10$ & $100 \pm 50$ & $100_{-60}$ & $180_{-30}^{+10}$ & $3.7 / 1642$ \\
\hline 2067 & $5.73 \pm 0.12$ & $0.62 \pm 0.05$ & $33 \pm 4$ & $175 \pm 25$ & $81 \pm 7$ & $121 \pm 6$ & $4.3 / 3602$ \\
\hline 2142 & $5.32 \pm 0.14$ & $0.65 \pm 0.02$ & $44 \pm 2$ & $172 \pm 10$ & $96 \pm 3$ & $114 \pm 7$ & $17 / 2702$ \\
\hline 2143 & $5.7 \pm 0.2$ & $0.64 \pm 0.01$ & $0^{+12}$ & $0^{+20}$ & $40 \pm 8$ & $108 \pm 6$ & $16 / 1392$ \\
\hline 2313 & $6.73 \pm 0.11$ & $0.63 \pm 0.01$ & $34 \pm 7$ & $0^{+20}$ & $40 \pm 5$ & $94 \pm 14$ & $3.6 / 2902$ \\
\hline 2320 & $7.04 \pm 0.13$ & $0.67 \pm 0.02$ & $37 \pm 7$ & $44_{-20}^{+15}$ & $38 \pm 5$ & $95 \pm 13$ & $4.5 / 2962$ \\
\hline 2426 & $6.65 \pm 0.10$ & $0.69 \pm 0.01$ & $55 \pm 4$ & $33_{-16}^{+10}$ & $42 \pm 5$ & $85 \pm 12$ & $5.2 / 4372$ \\
\hline 2550 & $6.63 \pm 0.15$ & $0.64 \pm 0.04$ & $26 \pm 5$ & $175 \pm 20$ & $80 \pm 6$ & $108 \pm 5$ & $7.5 / 2992$ \\
\hline 2681 & $8.41 \pm 0.08$ & $0.67 \pm 0.01$ & $48 \pm 3$ & $18_{-17}^{+10}$ & $33 \pm 4$ & $92 \pm 10$ & $6.0 / 6142$ \\
\hline 2685 & $8.11 \pm 0.10$ & $0.66 \pm 0.01$ & $54 \pm 4$ & $0^{-14}$ & $38 \pm 6$ & $113 \pm 12$ & $5.7 / 3256$ \\
\hline 3073 & $11.11 \pm 0.12$ & $0.67 \pm 0.01$ & $34 \pm 5$ & $46 \pm 14$ & $40 \pm 3$ & $93 \pm 8$ & $5.6 / 2662$ \\
\hline 3109 & $9.7 \pm 0.1$ & $0.64 \pm 0.02$ & $18_{-18}^{+10}$ & $0^{+15}$ & $42 \pm 2$ & $88 \pm 7$ & $17 / 1598$ \\
\hline 3178 & $11.71 \pm 0.09$ & $0.685 \pm 0.007$ & $45 \pm 2$ & $0^{+10}$ & $39 \pm 2$ & $103 \pm 7$ & $4 / 3442$ \\
\hline 3436 & $15.17 \pm 0.09$ & $0.705 \pm 0.005$ & $47 \pm 2$ & $24 \pm 11$ & $42 \pm 2$ & $95 \pm 4$ & $4.9 / 4337$ \\
\hline 3485 & $15.42 \pm 0.08$ & $0.707 \pm 0.005$ & $51 \pm 2$ & $1^{+10}$ & $43 \pm 2$ & $102 \pm 5$ & $5.2 / 4702$ \\
\hline 3512 & $15.43 \pm 0.12$ & $0.708 \pm 0.006$ & $53 \pm 2$ & $0^{+18}$ & $42 \pm 3$ & $94 \pm 6$ & $3.3 / 2632$ \\
\hline 3914 & $17.57 \pm 0.14$ & $0.694 \pm 0.007$ & $42 \pm 3$ & $0^{+10}$ & $38 \pm 3$ & $111 \pm 7$ & $2.4 / 1272$ \\
\hline 4013 & $19.09 \pm 0.10$ & $0.754 \pm 0.005$ & $46.4 \pm 1.4$ & $0^{+5}$ & $45.1 \pm 1.5$ & $104 \pm 4$ & $3.0 / 2830$ \\
\hline 4016 & $18.72 \pm 0.10$ & $0.745 \pm 0.006$ & $51 \pm 2$ & $0^{+5}$ & $46 \pm 2$ & $103 \pm 5$ & $3.1 / 2512$ \\
\hline 4220 & $20.20 \pm 0.09$ & $0.729 \pm 0.004$ & $43.4 \pm 1.3$ & $2_{-2}^{+13}$ & $37.6 \pm 1.3$ & $100 \pm 3$ & $2.2 / 2955$ \\
\hline 4268 & $21.78 \pm 0.13$ & $0.736 \pm 0.006$ & $40 \pm 2$ & $28 \pm 8$ & $38 \pm 2$ & $107 \pm 4$ & $7.3 / 3862$ \\
\hline 4372 & $22.94 \pm 0.10$ & $0.727 \pm 0.005$ & $37 \pm 2$ & $23 \pm 9$ & $37.4 \pm 1.5$ & $114 \pm 3$ & $3.6 / 3532$ \\
\hline 4577 & $23.89 \pm 0.14$ & $0.757 \pm 0.007$ & $40 \pm 2$ & $21 \pm 6$ & $39 \pm 2$ & $103 \pm 4$ & $7.0 / 3442$ \\
\hline 4584 & $25.23 \pm 0.07$ & $0.747 \pm 0.003$ & $42.0 \pm 1.0$ & $0^{+5}$ & $38.5 \pm 1.0$ & $109 \pm 2$ & $2.9 / 4222$ \\
\hline 4966 & $29.45 \pm 0.06$ & $0.764 \pm 0.002$ & $40.8 \pm 0.6$ & $0^{+5}$ & $39.5 \pm 0.6$ & $108.3 \pm 1.3$ & $1.3 / 50689$ \\
\hline 5011 & $32.97 \pm 0.07$ & $0.775 \pm 0.002$ & $44.1 \pm 0.7$ & $1_{-1}^{+5}$ & $40.1 \pm 0.6$ & $104.9 \pm 1.4$ & $1.4 / 50531$ \\
\hline 5387 & $34.11 \pm 0.08$ & $0.790 \pm 0.003$ & $41.4 \pm 0.8$ & $0^{+3}$ & $41.5 \pm 0.7$ & $107.8 \pm 1.5$ & $1.6 / 39604$ \\
\hline 5748 & $41.68 \pm 0.07$ & $0.811 \pm 0.002$ & $43.8 \pm 0.5$ & $0^{+2}$ & $40.2 \pm 0.5$ & $103.1 \pm 1.2$ & $1.3 / 38992$ \\
\hline 5810 & $42.46 \pm 0.07$ & $0.815 \pm 0.002$ & $42.9 \pm 0.5$ & $1_{-1}^{+4}$ & $42.8 \pm 0.6$ & $117.4 \pm 1.0$ & $1.6 / 46012$ \\
\hline 6003 & $46.50 \pm 0.07$ & $0.815 \pm 0.002$ & $39.6 \pm 0.5$ & $0^{+1}$ & $38.4 \pm 0.5$ & $101.2 \pm 1.1$ & $1.6 / 44992$ \\
\hline 6129 & $52.82 \pm 0.08$ & $0.833 \pm 0.002$ & $42.7 \pm 0.5$ & $0^{+2}$ & $42.1 \pm 0.4$ & $107.7 \pm 1.1$ & $1.3 / 46012$ \\
\hline 6170 & $54.05 \pm 0.08$ & $0.831 \pm 0.002$ & $42.2 \pm 0.5$ & $0^{+2}$ & $38.8 \pm 0.4$ & $107.8 \pm 1.1$ & $1.5 / 43672$ \\
\hline 6283 & $53.63 \pm 0.07$ & $0.829 \pm 0.001$ & $39.6 \pm 0.4$ & $0^{+2}$ & $38.8 \pm 0.4$ & $107.7 \pm 0.9$ & $1.5 / 44842$ \\
\hline 6605 & $61.36 \pm 0.10$ & $0.843 \pm 0.002$ & $38.2 \pm 0.5$ & $0^{+2}$ & $39.1 \pm 0.5$ & $109.0 \pm 1.1$ & $2.9 / 42892$ \\
\hline 6693 & $62.69 \pm 0.08$ & $0.858 \pm 0.001$ & $43.0 \pm 0.4$ & $0^{+3}$ & $35.9 \pm 0.4$ & $101.5 \pm 1.0$ & $1.5 / 38992$ \\
\hline 6973 & $73.81 \pm 0.08$ & $0.880 \pm 0.001$ & $44.6 \pm 0.3$ & $0^{+2}$ & $42.1 \pm 0.4$ & $117.4 \pm 0.7$ & $1.5 / 40357$ \\
\hline 7085 & $77.19 \pm 0.08$ & $0.872 \pm 0.001$ & $39.3 \pm 0.3$ & $0^{+1}$ & $39.8 \pm 0.3$ & $111.8 \pm 0.6$ & $1.5 / 29112$ \\
\hline 7228 & $82.51 \pm 0.08$ & $0.874 \pm 0.001$ & $40.0 \pm 0.3$ & $0^{+1}$ & $39.4 \pm 0.3$ & $109.1 \pm 0.7$ & $1.4 / 35677$ \\
\hline 7620 & $93.61 \pm 0.09$ & $0.893 \pm 0.001$ & $42.7 \pm 0.3$ & $0^{+4}$ & $38.9 \pm 0.3$ & $105.1 \pm 0.6$ & $1.3 / 42892$ \\
\hline 7730 & $98.98 \pm 0.08$ & $0.8905 \pm 0.0008$ & $36.2 \pm 0.2$ & $0^{+1}$ & $36.3 \pm 0.2$ & $109.4 \pm 0.5$ & $1.6 / 40942$ \\
\hline 7901 & $107.73 \pm 0.08$ & $0.8916 \pm 0.0007$ & $35.8 \pm 0.2$ & $0^{+2}$ & $35.9 \pm 0.2$ & $109.1 \pm 0.4$ & $1.5 / 44452$ \\
\hline 8139 & $121.59 \pm 0.08$ & $0.9095 \pm 0.0006$ & $37.6 \pm 0.1$ & $0^{+4}$ & $32.0 \pm 0.1$ & $104.4 \pm 0.4$ & $0.5 / 278820$ \\
\hline 8370 & $128.07 \pm 0.08$ & $0.9142 \pm 0.0006$ & $36.6 \pm 0.2$ & $0^{+1}$ & $33.4 \pm 0.2$ & $105.4 \pm 0.4$ & $0.8 / 254302$ \\
\hline 8448 & $132.59 \pm 0.07$ & $0.9109 \pm 0.0008$ & $33.5 \pm 0.2$ & $12 \pm 3$ & $28.7 \pm 0.1$ & $103.4 \pm 0.4$ & $0.9 / 301177$ \\
\hline 8737 & $142.25 \pm 0.07$ & $0.9185 \pm 0.0005$ & $32.8 \pm 0.1$ & $0^{+2}$ & $29.8 \pm 0.1$ & $104.2 \pm 0.4$ & $0.6 / 251707$ \\
\hline 8824 & $136.58 \pm 0.08$ & $0.9169 \pm 0.0008$ & $28.4 \pm 0.2$ & $1_{-1}^{+3}$ & $26.9 \pm 0.1$ & $105.7 \pm 0.4$ & $0.7 / 241327$ \\
\hline 9089 & $155.92 \pm 0.06$ & $0.9251 \pm 0.0005$ & $30.5 \pm 0.1$ & $0^{+1}$ & $25.8 \pm 0.1$ & $102.3 \pm 0.4$ & $1.1 / 265987$ \\
\hline 9233 & $161.00 \pm 0.06$ & $0.9290 \pm 0.0006$ & $28.8 \pm 0.1$ & $1_{-1}^{+2}$ & $25.8 \pm 0.1$ & $102.4 \pm 0.3$ & $1.0 / 301177$ \\
\hline 9321 & $165.62 \pm 0.05$ & $0.9304 \pm 0.0003$ & $29.1 \pm 0.1$ & $0^{+1}$ & $23.5 \pm 0.1$ & $98.4 \pm 0.3$ & $0.9 / 284272$ \\
\hline 9509 & $168.68 \pm 0.05$ & $0.9467 \pm 0.0003$ & $31.7 \pm 0.1$ & $0^{+1}$ & $26.7 \pm 0.1$ & $97.8 \pm 0.2$ & $1.4 / 288550$ \\
\hline 9568 & $176.01 \pm 0.04$ & $0.9378 \pm 0.0002$ & $28.6 \pm 0.1$ & $0^{+1}$ & $18.4 \pm 0.1$ & $111.6 \pm 0.3$ & $1.3 / 283341$ \\
\hline
\end{tabular}

Notes. ${ }^{\text {a }}$ Before 2000, all 26 frequency channels in the data were averaged into one band of effective bandwidth $208 \mathrm{MHz}$ to boost the signal; between 2000 and 2009, 26 Hanning-smoothed channels, each of width $8 \mathrm{MHz}$ were used in the fit; since mid-2009, after the installation of the Compact Array Broadband Backend (CABB), 208 channels in the same frequency range were extracted, each of width $1 \mathrm{MHz}$. Since 2012, the ATCA sensitivity has improved by $\sim 40 \%$ as a result of the installation of new receivers.

size between days 6000 and 7500 , and it is larger after day 7500 . Over time, $R_{1}$ increases linearly without obvious deceleration. In contrast, $R_{2}$ exhibits a break around day 7000 , as did the torus radius. This results in a decrease in the ratio $R_{2} / R_{1}$, which shows a remarkably similar trend to that of $\theta$ (Figure 4(c)). The ring and torus fits give nearly identical flux densities and both suggest a decreasing trend in the degree of asymmetry since day $\sim 7500$. For completeness, we also show in Figure 5 the best-fit $R_{1}$ and $R_{2}$ from the ring fitting with a fixed aspect ratio. We obtained larger $R_{1}$ and smaller $R_{2}$ as compared to the ring fit with varying aspect ratio, and there is a hint of a break in the expansion around day 7500 . 
Table 3

Best-fit Parameters for the Ring Model with Statistical Uncertainties at a 68\% Confidence Level

\begin{tabular}{|c|c|c|c|c|c|c|}
\hline Day & $\begin{array}{l}\text { Flux } \\
(\mathrm{mJy})\end{array}$ & $\begin{array}{l}\text { Semi-major } \\
\text { Axis (") }\end{array}$ & $\begin{array}{l}\text { Semi-minor } \\
\text { Axis (") }\end{array}$ & $\begin{array}{c}\text { Asymmetry } \\
(\%)\end{array}$ & $\begin{array}{c}\phi \\
\left({ }^{\circ}\right)\end{array}$ & $\chi_{v}^{2} / \mathrm{dof}^{\mathrm{a}}$ \\
\hline 1786 & $3.70 \pm 0.12$ & $0.55 \pm 0.03$ & $0.50 \pm 0.03$ & $33 \pm 16$ & $141 \pm 26$ & $1.8 / 2108$ \\
\hline 1852 & $3.59 \pm 0.14$ & $0.53 \pm 0.04$ & $0.48 \pm 0.04$ & $25 \pm 17$ & $124 \pm 46$ & $3.7 / 1643$ \\
\hline 2067 & $5.17 \pm 0.09$ & $0.57 \pm 0.02$ & $0.49 \pm 0.01$ & $27 \pm 5$ & $105 \pm 16$ & $4.4 / 3603$ \\
\hline 2142 & $4.84 \pm 0.11$ & $0.53 \pm 0.02$ & $0.51 \pm 0.02$ & $17 \pm 8$ & $132 \pm 29$ & $17 / 2703$ \\
\hline 2143 & $5.62 \pm 0.14$ & $0.63 \pm 0.03$ & $0.44 \pm 0.02$ & $39 \pm 8$ & $94 \pm 24$ & $16 / 1393$ \\
\hline 2313 & $6.68 \pm 0.09$ & $0.57 \pm 0.02$ & $0.44 \pm 0.01$ & $34 \pm 5$ & $90 \pm 15$ & $3.6 / 2903$ \\
\hline 2320 & $6.87 \pm 0.10$ & $0.61 \pm 0.02$ & $0.47 \pm 0.01$ & $31 \pm 4$ & $92 \pm 13$ & $4.5 / 2963$ \\
\hline 2426 & $6.47 \pm 0.08$ & $0.56 \pm 0.01$ & $0.50 \pm 0.01$ & $28 \pm 4$ & $86 \pm 9$ & $5.2 / 4373$ \\
\hline 2550 & $6.12 \pm 0.12$ & $0.58 \pm 0.02$ & $0.54 \pm 0.02$ & $32 \pm 6$ & $117 \pm 12$ & $7.5 / 2993$ \\
\hline 2681 & $8.23 \pm 0.06$ & $0.58 \pm 0.01$ & $0.48 \pm 0.01$ & $26 \pm 3$ & $87 \pm 8$ & $6.0 / 6143$ \\
\hline 2685 & $7.94 \pm 0.08$ & $0.56 \pm 0.01$ & $0.49 \pm 0.01$ & $29 \pm 4$ & $109 \pm 10$ & $5.7 / 3257$ \\
\hline 3073 & $10.86 \pm 0.10$ & $0.62 \pm 0.01$ & $0.47 \pm 0.01$ & $33 \pm 2$ & $87 \pm 8$ & $5.6 / 2663$ \\
\hline 3109 & $9.73 \pm 0.23$ & $0.60 \pm 0.01$ & $0.50 \pm 0.01$ & $36 \pm 8$ & $92 \pm 32$ & $17 / 1599$ \\
\hline 3178 & $11.50 \pm 0.07$ & $0.601 \pm 0.007$ & $0.487 \pm 0.006$ & $32 \pm 2$ & $98 \pm 6$ & $4.0 / 3443$ \\
\hline 3436 & $14.76 \pm 0.07$ & $0.607 \pm 0.005$ & $0.495 \pm 0.004$ & $33 \pm 1$ & $94 \pm 4$ & $5.0 / 4338$ \\
\hline 3485 & $15.07 \pm 0.07$ & $0.598 \pm 0.006$ & $0.510 \pm 0.004$ & $33 \pm 2$ & $98 \pm 4$ & $5.2 / 4703$ \\
\hline 3512 & $15.06 \pm 0.10$ & $0.592 \pm 0.007$ & $0.508 \pm 0.005$ & $32 \pm 2$ & $91 \pm 5$ & $3.4 / 2633$ \\
\hline 3914 & $17.31 \pm 0.12$ & $0.624 \pm 0.006$ & $0.491 \pm 0.007$ & $33 \pm 3$ & $107 \pm 7$ & $2.4 / 1273$ \\
\hline 4013 & $18.53 \pm 0.09$ & $0.646 \pm 0.005$ & $0.527 \pm 0.004$ & $35.5 \pm 1.2$ & $99 \pm 3$ & $3.0 / 2831$ \\
\hline 4016 & $18.36 \pm 0.09$ & $0.632 \pm 0.005$ & $0.545 \pm 0.006$ & $35.8 \pm 1.3$ & $98 \pm 4$ & $3.1 / 2513$ \\
\hline 4220 & $19.76 \pm 0.07$ & $0.643 \pm 0.004$ & $0.509 \pm 0.003$ & $31.4 \pm 1.0$ & $96 \pm 3$ & $2.2 / 2956$ \\
\hline 4268 & $21.19 \pm 0.11$ & $0.656 \pm 0.006$ & $0.506 \pm 0.005$ & $31.1 \pm 1.5$ & $104 \pm 4$ & $7.4 / 3863$ \\
\hline 4372 & $22.43 \pm 0.08$ & $0.663 \pm 0.004$ & $0.500 \pm 0.004$ & $32.1 \pm 1.2$ & $111 \pm 3$ & $3.6 / 3533$ \\
\hline 4577 & $23.26 \pm 0.12$ & $0.674 \pm 0.006$ & $0.515 \pm 0.005$ & $32.7 \pm 1.4$ & $98 \pm 4$ & $7.1 / 3443$ \\
\hline 4584 & $24.69 \pm 0.07$ & $0.663 \pm 0.003$ & $0.521 \pm 0.003$ & $31.6 \pm 0.8$ & $103 \pm 2$ & $2.9 / 4223$ \\
\hline 4966 & $28.75 \pm 0.05$ & $0.681 \pm 0.002$ & $0.527 \pm 0.002$ & $32.9 \pm 0.5$ & $102 \pm 1$ & $1.3 / 50690$ \\
\hline 5011 & $32.03 \pm 0.06$ & $0.677 \pm 0.002$ & $0.531 \pm 0.002$ & $32.7 \pm 0.5$ & $100 \pm 1$ & $1.4 / 50532$ \\
\hline 5387 & $33.19 \pm 0.07$ & $0.694 \pm 0.002$ & $0.547 \pm 0.002$ & $34.0 \pm 0.5$ & $102 \pm 1$ & $1.6 / 39605$ \\
\hline 5748 & $40.56 \pm 0.06$ & $0.710 \pm 0.002$ & $0.564 \pm 0.001$ & $33.0 \pm 0.4$ & $97 \pm 1$ & $1.3 / 38993$ \\
\hline 5810 & $41.24 \pm 0.06$ & $0.723 \pm 0.002$ & $0.568 \pm 0.002$ & $34.8 \pm 0.4$ & $110 \pm 1$ & $1.6 / 46013$ \\
\hline 6003 & $45.35 \pm 0.06$ & $0.723 \pm 0.002$ & $0.561 \pm 0.002$ & $32.2 \pm 0.4$ & $94 \pm 1$ & $1.6 / 44993$ \\
\hline 6129 & $51.43 \pm 0.07$ & $0.733 \pm 0.002$ & $0.579 \pm 0.002$ & $34.7 \pm 0.3$ & $100 \pm 1$ & $1.3 / 46013$ \\
\hline 6170 & $52.63 \pm 0.07$ & $0.734 \pm 0.001$ & $0.577 \pm 0.002$ & $32.1 \pm 0.3$ & $102 \pm 1$ & $1.5 / 43673$ \\
\hline 6283 & $52.18 \pm 0.06$ & $0.743 \pm 0.001$ & $0.569 \pm 0.001$ & $32.8 \pm 0.3$ & $101 \pm 1$ & $1.5 / 44843$ \\
\hline 6605 & $59.86 \pm 0.09$ & $0.758 \pm 0.002$ & $0.586 \pm 0.002$ & $33.0 \pm 0.4$ & $101 \pm 1$ & $2.9 / 42893$ \\
\hline 6693 & $61.21 \pm 0.07$ & $0.7622 \pm 0.0013$ & $0.5979 \pm 0.0013$ & $30.1 \pm 0.3$ & $95 \pm 1$ & $1.5 / 38993$ \\
\hline 6973 & $71.33 \pm 0.07$ & $0.7743 \pm 0.0011$ & $0.6118 \pm 0.0014$ & $33.7 \pm 0.3$ & $110 \pm 1$ & $1.5 / 40358$ \\
\hline 7085 & $74.60 \pm 0.07$ & $0.7695 \pm 0.0012$ & $0.5939 \pm 0.0011$ & $32.6 \pm 0.2$ & $103 \pm 1$ & $1.6 / 29113$ \\
\hline 7228 & $80.53 \pm 0.07$ & $0.7825 \pm 0.0009$ & $0.6171 \pm 0.0010$ & $33.0 \pm 0.2$ & $101 \pm 1$ & $1.4 / 35678$ \\
\hline 7620 & $90.47 \pm 0.08$ & $0.7830 \pm 0.0011$ & $0.6208 \pm 0.0009$ & $31.8 \pm 0.2$ & $97 \pm 1$ & $1.4 / 42893$ \\
\hline 7730 & $96.36 \pm 0.07$ & $0.8058 \pm 0.0008$ & $0.6179 \pm 0.0008$ & $30.9 \pm 0.2$ & $102 \pm 1$ & $1.6 / 40943$ \\
\hline 7901 & $104.94 \pm 0.06$ & $0.8075 \pm 0.0007$ & $0.6220 \pm 0.0007$ & $30.8 \pm 0.1$ & $102 \pm 0$ & $1.5 / 44453$ \\
\hline 8139 & $117.73 \pm 0.07$ & $0.8241 \pm 0.0006$ & $0.6263 \pm 0.0006$ & $27.7 \pm 0.1$ & $97 \pm 0$ & $0.5 / 278821$ \\
\hline 8370 & $124.86 \pm 0.07$ & $0.8243 \pm 0.0006$ & $0.6428 \pm 0.0007$ & $28.6 \pm 0.1$ & $98 \pm 0$ & $0.8 / 254303$ \\
\hline 8448 & $129.50 \pm 0.06$ & $0.8406 \pm 0.0005$ & $0.6282 \pm 0.0006$ & $25.6 \pm 0.1$ & $94 \pm 0$ & $0.9 / 301178$ \\
\hline 8737 & $139.17 \pm 0.06$ & $0.8433 \pm 0.0005$ & $0.6430 \pm 0.0005$ & $26.2 \pm 0.1$ & $96 \pm 0$ & $0.7 / 251708$ \\
\hline 8824 & $133.95 \pm 0.07$ & $0.8582 \pm 0.0006$ & $0.6373 \pm 0.0007$ & $24.1 \pm 0.1$ & $96 \pm 0$ & $0.7 / 241328$ \\
\hline 9089 & $153.09 \pm 0.05$ & $0.8593 \pm 0.0004$ & $0.6438 \pm 0.0005$ & $23.1 \pm 0.1$ & $92 \pm 0$ & $1.1 / 265988$ \\
\hline 9233 & $157.94 \pm 0.05$ & $0.8698 \pm 0.0004$ & $0.6401 \pm 0.0005$ & $23.4 \pm 0.1$ & $93 \pm 0$ & $1.1 / 301178$ \\
\hline 9321 & $162.98 \pm 0.05$ & $0.8703 \pm 0.0003$ & $0.6527 \pm 0.0004$ & $21.5 \pm 0.1$ & $88 \pm 0$ & $0.9 / 284273$ \\
\hline 9509 & $165.10 \pm 0.04$ & $0.8761 \pm 0.0003$ & $0.6583 \pm 0.0003$ & $24.0 \pm 0.1$ & $88 \pm 0$ & $1.4 / 288551$ \\
\hline 9568 & $173.03 \pm 0.04$ & $0.8818 \pm 0.0002$ & $0.6574 \pm 0.0003$ & $16.2 \pm 0.1$ & $100 \pm 0$ & $1.4 / 283342$ \\
\hline
\end{tabular}

Notes. ${ }^{\text {a }}$ Before 2000, all 26 frequency channels in the data were averaged into one band of effective bandwidth $208 \mathrm{MHz}$ to boost the signal; between 2000 and 2009, 26 Hanning-smoothed channels, each of width $8 \mathrm{MHz}$ were used in the fit; since mid-2009, after the installation of the Compact Array Broadband Backend (CABB), 208 channels in the same frequency range were extracted, each of width $1 \mathrm{MHz}$. Since 2012, the ATCA sensitivity has improved by $\sim 40 \%$ as a result of the installation of new receivers.

\section{RATE OF EXPANSION}

The torus fitting results shown in Figure 4(b) suggest a possible deceleration of the remnant. We therefore followed Racusin et al. (2009) to fit the radius evolution with a broken linear function. Only observations after day 4200 were used here, since we are most interested in the late evolution of the remnant. We note that the radius measurements in Table 2 have statistical uncertainties of the order of $0{ }^{\prime} .001$, much smaller than the scatter from measurement to measurement. We therefore added in quadrature systematic uncertainties of $0{ }^{\prime} 005$ to the measurements errors. This value was chosen to give a reduced 

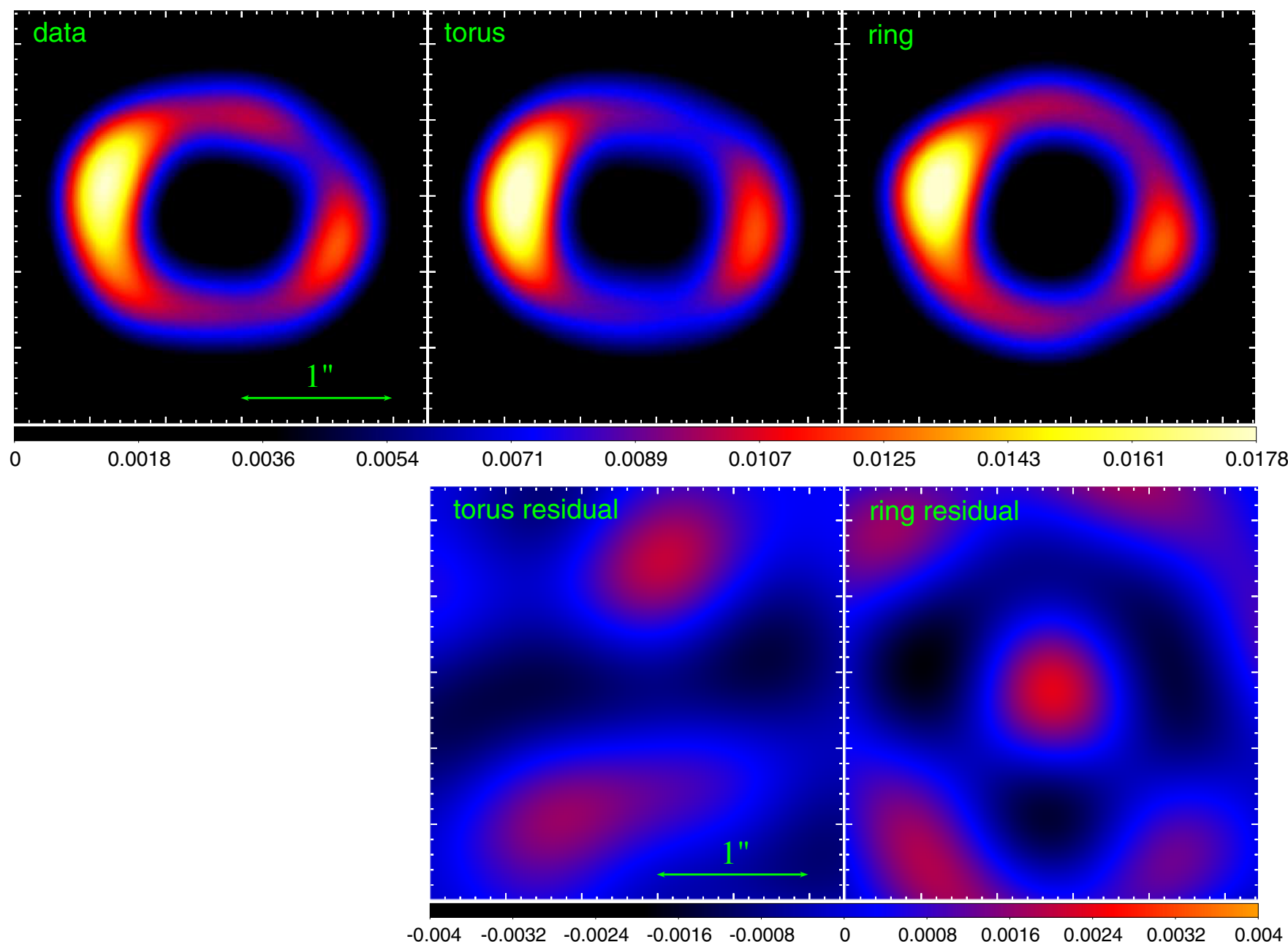

Figure 3. Radio image of SN 1987A at $9 \mathrm{GHz}$ taken on 2012 September 1 (left) compared with images of the best-fit torus (middle) and elliptical ring (right) models. The lower panels show the maps of the residual (i.e., data minus model) visibilities for which no deconvolution has been applied. Note that the upper and lower panels have different color scales.

(A color version of this figure is available in the online journal.)

$\chi^{2}$ value of about 1 for the broken linear fit. We have tried different values from 0 to 0 .'05 and confirm that our results are independent of the choice.

Table 4 lists the best-fit expansion velocity and transition day. Uncertainties quoted are $68 \%$ confidence intervals determined using a bootstrapping technique (Efron \& Tibshirani 1993) with 10,000 simulations. The break in the expansion of the torus model occurred on day $7000_{-100}^{+200}$, and was the same as $R_{2}$ of the ring model (day $7000 \pm 300$ ). However, $R_{1}$ shows no breaks and a simple linear fit is statistically preferred. Finally, the ring fit with a constant aspect ratio suggests a somewhat later transition at day $7600 \pm 200$. At a source distance of $51.4 \mathrm{kpc}$ (Panagia 1999), the torus fits suggest expansion velocities of $v_{1}=$ $4600_{-200}^{+150} \mathrm{~km} \mathrm{~s}^{-1}$ and $v_{2}=2400_{-200}^{+100} \mathrm{~km} \mathrm{~s}^{-1}$ before and after the break, respectively. While $v_{1}$ is larger than the expansion rate $3890 \pm 50 \mathrm{~km} \mathrm{~s}^{-1}$ of $R_{1}$, both values are consistent with the result of $4000 \pm 400 \mathrm{~km} \mathrm{~s}^{-1}$ reported by N08. Similar to the torus radius, the expansion velocity of $R_{2}$ decreased by nearly $50 \%$, from $3300 \mathrm{~km} \mathrm{~s}^{-1}$ to $1750 \mathrm{~km} \mathrm{~s}^{-1}$, after the break.

\section{DISCUSSION}

Non-thermal radio emission from SNRs traces the particle acceleration in shocks. Theories suggest that the emission is generally distributed between the forward and reverse shocks (e.g., Jun \& Norman 1996). When the shocks of SN 1987A travel outward and encounter the equatorial ring, we expect the radio-emitting region to have a complex structure in $3 \mathrm{D}$ (e.g., Suzuki et al. 1993; Luo et al. 1994; Blondin et al. 1996), with components from both high-latitude material above the equatorial plane and the ring itself. To characterize the remnant geometry, we have carried out Fourier modeling using a truncated-shell torus model and an elliptical thin ring model. Although both provide adequate fits to the data, we should note that these models are simple parameterizations of the remnant structure, and that the geometry inferred from the fitting is model-dependent and could be systematically biased. For instance, if the actual shape of the emission resembles a crescent torus (Plait et al. 1995), then the radius measurement obtained from our torus model would depend sensitively on $\theta$.

One major discrepancy between the torus and ring models is the radius measurement. We find that the radius obtained from fitting a torus model is always larger than that from a ring model. This could be a projection effect due to differences in the model geometry or the emission at high latitudes, to which the torus model is more sensitive, being physically further away from the center. For the former, consider a thin spherical shell in 3D: in the image the emission would appear to peak inside the shell 

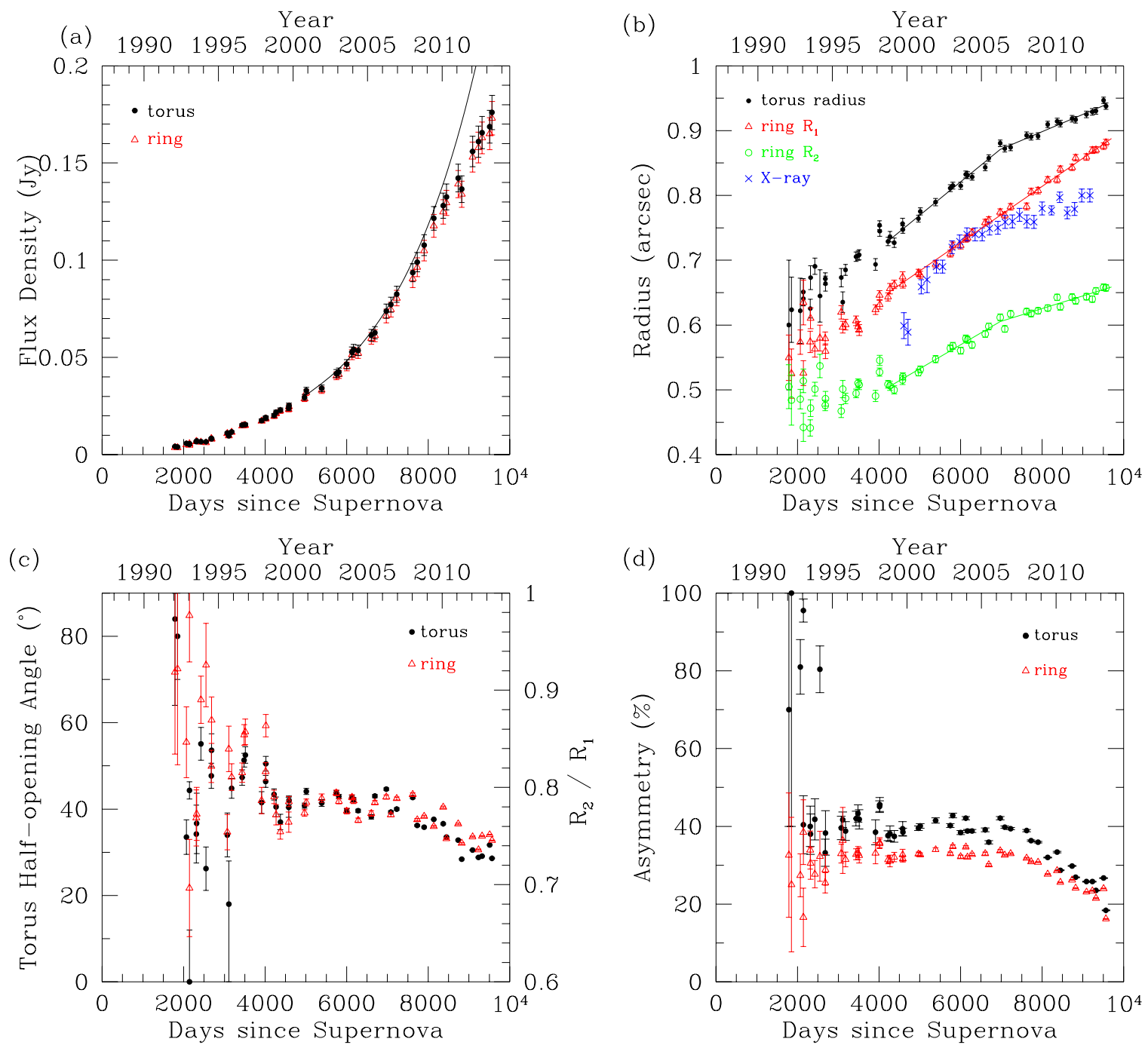

Figure 4. (a) Model flux densities at $9 \mathrm{GHz}$. Systematic uncertainties of 5\% (Zanardo et al. 2013) are combined with the statistical uncertainties at a 68\% confidence level. The solid line is the exponential fit from Zanardo et al. (2010). (b) Best-fit radius of the torus model and semi-major $\left(R_{1}\right)$ and semi-minor $\left(R_{2}\right)$ axes of the ring model, compared with the X-ray radius reported by Helder et al. (2013). Systematic uncertainties of 0.'005 are combined with the statistical uncertainties at $68 \%$ confidence level. The solid lines are the best-fit expansion rates in Table 4. (c) Best-fit torus half-opening angle $\theta$ compared with the ratio $R_{2} / R_{1}$ of the ring model. (d) Best-fit asymmetry in the surface brightness.

(A color version of this figure is available in the online journal.)

Table 4

Expansion of the Radio Remnant of SN 1987A Since Day 4200, with Uncertainties at a 68\% Confidence Level

\begin{tabular}{|c|c|c|c|}
\hline Model & Transition Day & $\begin{array}{c}v_{1} \\
\left(\mathrm{~km} \mathrm{~s}^{-1}\right)\end{array}$ & $\begin{array}{c}v_{2} \\
\left(\mathrm{~km} \mathrm{~s}^{-1}\right)\end{array}$ \\
\hline Torus & $7000_{-100}^{+200}$ & $4600_{-200}^{+150}$ & $2400_{-200}^{+100}$ \\
\hline Ring semi-major axis ${ }^{\mathrm{a}}$ & $\ldots$ & $3890 \pm 50$ & $\ldots$ \\
\hline Ring semi-minor axis & $7000 \pm 300$ & $3300 \pm 200$ & $1750_{-300}^{+150}$ \\
\hline Ring semi-major axis (aspect ratio fixed) ${ }^{b}$ & $7600 \pm 200$ & $3940 \pm 70$ & $2900 \pm 100$ \\
\hline Ring semi-minor axis (aspect ratio fixed) ${ }^{\mathrm{b}}$ & $7600 \pm 200$ & $2710 \pm 50$ & $2000_{-70}^{+60}$ \\
\hline
\end{tabular}

Notes.

a A simple linear expansion is preferred over a broken linear fit.

${ }^{\mathrm{b}}$ Expansion of the semi-major and semi-minor axes are linked as the ring's aspect ratio is fixed during the fit.

radius because of projection and the finite spatial resolution of the telescope. Hence, a 3D shell model would require a larger radius compared to a simple thin ring model. Our torus model varies between a shell and a ring depending on $\theta$ (see Figure 2). The projection effect is minimum at $\theta=0$, where the torus reduces to a 2D ring, and increases with $\theta$. Given that the bestfit $\theta$ is significantly greater than zero, the above discrepancy is not unexpected. Alternatively, the radio-emitting region could have a larger radius at higher latitudes than at the equatorial plane because the SN shock travels at a larger velocity in the 


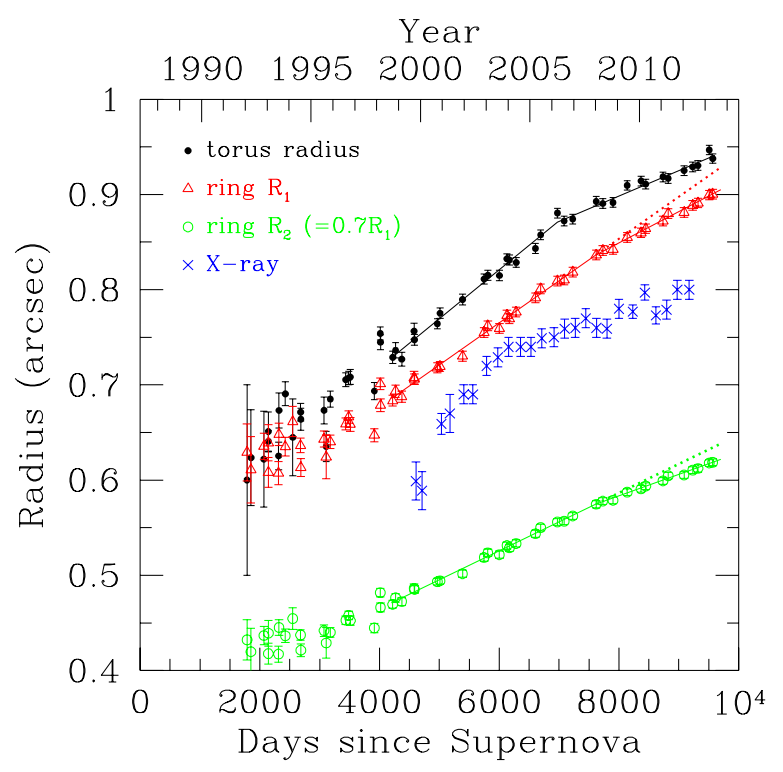

Figure 5. Same as Figure 4(b), but the aspect ratio of the ring model is fixed at $R_{2} / R_{1}=0.7$ according to the inclination of the system. To better illustrate the break in the expansion of $R_{1}$ and $R_{2}$, the dotted lines indicate the expansion without any breaks for a direct comparison.

(A color version of this figure is available in the online journal.)

low-density environment (Blondin et al. 1996). If this is the case, any 3D models sensitive to high-latitude emission, such as the torus model, will tend to give a larger radius. It is worth noting that in both scenarios above, there is a possible coupling between the torus radius and $\theta$, and it could correspondingly impact the expansion measurement.

In previous studies, the reported radii of SNR 1987A in radio and X-rays show a 10\% difference over day 5000-8000 (N08; Racusin et al. 2009). It was first pointed out by Gaensler et al. (2007) that the discrepancy may not be physical but due to different measuring techniques. This idea is similar to what we have discussed above. In particular, measurements from N08 were made with a torus model, while the X-ray results were obtained from ring fitting (Racusin et al. 2009; Helder et al. 2013). Gaensler et al. (2007) analyzed both radio and X-ray data taken in 2004 (day 6300) using a consistent method and showed that the SNR sizes agree to within $1 \%$. Our results confirm their finding: $R_{1}$ from the ring model at this epoch is fully consistent with the X-ray radius reported by Helder et al. (2013; see Figure 4(b)).

More generally, the ring fits allow a meaningful comparison between the radio and X-ray SNR radii. From Figure 4(b), the radius of the radio remnant has exceeded that of the $\mathrm{X}$-ray counterpart since day $\sim 7500$. This is also supported by the torus fits: $\mathrm{Ng}$ et al. (2009) employed the same torus model as ours to fit the X-ray data taken on 2008 April (day 7736), and found that it provides a slightly better fit than a simple ring, with a radius of $00^{\prime} .82 \pm 00^{\prime} .02$ and $\theta=26^{\circ} \pm 3^{\circ}$. Both values are smaller than what we have obtained for the radio remnant at the same epoch ( $0^{\prime} .89$ and 36.2 , respectively; Table 2$)$, suggesting a smaller extent of the remnant in X-rays than in radio. This agrees with the simulation results (Jun \& Norman 1996). In addition, the radio emission may partly originate from fast shocks at high latitudes that have not yet decelerated. This scenario can help explain the fact that the radio remnant has apparently expanded beyond the optical inner ring with radius $\sim 00^{\prime \prime} 85$ (Plait et al. 1995).
Figure 4(c) shows that $\theta$ started to decrease at day $\sim 7000$, as did the ratio $R_{2} / R_{1}$ of the ring model. The results suggest that the radio emission from lower latitudes has gradually dominated, which could be a consequence of shock interaction with the dense circumstellar medium in the equatorial ring. If the contribution of radio emission from high latitudes continues to diminish, then we would expect the radius estimates from both the torus and ring models to converge eventually.

The morphological evolution of SNR 1987A was accompanied by a reduction in the degree of surface brightness asymmetry. Since the radio remnant first emerged, the eastern lobe has always been brighter than the western one (see Figure 1), and this has been attributed to faster shocks in the east (e.g., Gaensler et al. 1997; N08). This scenario is supported by the significantly higher expansion velocities of the eastern lobe measured from radio observations at higher frequencies (Zanardo et al. 2013), and by the higher shock temperature found in the east from X-ray studies (Zhekov et al. 2009). The faster shock in the east is expected to encounter the equatorial ring, slow down, and exit the ring earlier than in the west. This would reduce the radio emissivity in the eastern rim and hence the overall brightness asymmetry. If the observed trend continues, the western hemisphere of the radio remnant may become brighter than the eastern hemisphere in a few years, as predicted by 3D simulations of the expanding remnant (T. M. Potter et al. 2013, in preparation). The same picture could also be applied to the X-ray emission, which exhibits similar variations in the projected brightness distribution ( $\mathrm{Ng}$ et al. 2011b).

In X-rays, Helder et al. (2013) reported drastic deceleration of the SNR from $8500 \mathrm{~km} \mathrm{~s}^{-1}$ to $1820 \mathrm{~km} \mathrm{~s}^{-1}$ on day $\sim 5900$. However, we do not find conclusive evidence for a similar deceleration in the radio emission. In particular, $R_{1}$ from the ring fit shows a constant expansion at $3890 \mathrm{~km} \mathrm{~s}^{-1}$. For the torus fit, while Figure 4(b) suggests a break in the expansion, the coupling between the radius and $\theta$ described above makes the interpretation difficult. If the apparent break is physical, it would well match the deceleration of the reverse shock predicted by one-dimensional hydrodynamic simulations (see Figure 9 of Dewey et al. 2012). However, we believe that the break is likely caused by the decreasing trend in $\theta$. Hence, it reflects a change of the emission geometry rather than the slowing down of the shock. Observing a deceleration of $R_{1}$ in future will confirm this picture. This also gives a prediction that once $\theta$ stops shrinking, the torus radius expansion rate should return to the same value as that measured for $R_{1}$.

\section{CONCLUSION}

We have studied the evolution of the radio remnant of SN 1987A using ATCA $9 \mathrm{GHz}$ imaging observations taken between 1992 and 2013 and have carried out Fourier modeling on the visibility data to quantitatively measure the remnant structure. A truncated-shell torus model and an elliptical ring model were used to fit the remnant morphology. They both suggest a gradual decrease in the latitude extent of the remnant starting from day $\sim 7000$, implying that the radio emission from the equatorial region has progressively dominated. This has been accompanied by a decreasing trend in the brightness asymmetry in the east-west direction. Together these could indicate a new stage of the remnant evolution, such that the forward shock has fully engulfed the entire inner ring and is now interacting with the densest part of the circumstellar medium.

As a direct comparison between the torus and ring model fits, they give similar results for most parameters, but the former 
always suggests a larger radius. The discrepancy could be attributed to the projection effect or emission at high latitudes to which the torus model is more sensitive. This also leads to different expansion measurements. Although the torus fit shows a break in the expansion around day 7000 with the velocity slowing down from $4600 \mathrm{~km} \mathrm{~s}^{-1}$ to $2400 \mathrm{~km} \mathrm{~s}^{-1}$, the ring fit indicates a constant expansion rate of $3890 \mathrm{~km} \mathrm{~s}^{-1}$. We argue that the apparent break could be the result of coupling between the torus radius and opening angle. We expect in the future when the latter stays constant, both the torus and ring fits should give consistent expansion velocity. Further observations at higher resolution (with VLBI or ALMA, for example) would be useful in understanding the true 3D nature of the evolving remnant, and the time and latitude dependence of the expansion velocity.

We thank the referee for useful suggestions. The Australia Telescope Compact Array is part of the Australia Telescope, which is funded by the Commonwealth of Australia for operation as a National Facility managed by CSIRO. Parts of this research were conducted by the Australian Research Council Centre of Excellence for All-sky Astrophysics (CAASTRO) through project number CE110001020.

Facility: ATCA

\section{REFERENCES}

Blondin, J. M., Lundqvist, P., \& Chevalier, R. A. 1996, ApJ, 472, 257

Burrows, C. J., Krist, J., Hester, J. J., et al. 1995, ApJ, 452, 680

Dewey, D., Dwarkadas, V. V., Haberl, F., Sturm, R., \& Canizares, C. R. 2012, ApJ, 752, 103

Efron, B., \& Tibshirani, R. J. 1993, An Introduction to the Bootstrap (New York: Chapman and Hall)

Gaensler, B. M., Manchester, R. N., Staveley-Smith, L., et al. 1997, ApJ, 479,845

Gaensler, B. M., Staveley-Smith, L., Manchester, R. N., et al. 2007, in AIP Conf. Proc. 937, Supernova 1987A: 20 Years After: Supernovae and Gamma-Ray Bursters, ed. S. Immler, K. Weiler, \& R. McCray (Melville, NY: AIP), 86

Gull, S. F., \& Daniell, G. J. 1978, Natur, 272, 686

Helder, E. A., Broos, P. S., Dewey, D., et al. 2013, ApJ, 764, 11
Immler, S., Weiler, K., \& McCray, R. (eds.) 2007, in AIP Conf. Proc. 937, Supernova 1987A: 20 Years After: Supernovae and Gamma-Ray Bursters (Melville, NY: AIP)

Jun, B.-I., \& Norman, M. L. 1996, ApJ, 465, 800

Kemball, A., \& Martinsek, A. 2005, AJ, 129, 1760

Lakićević, M., Zanardo, G., van Loon, J. T., et al. 2012, A\&A, 541, L2

Luo, D., McCray, R., \& Slavin, J. 1994, ApJ, 430, 264

Manchester, R. N., Gaensler, B. M., Staveley-Smith, L., Kesteven, M. J., \& Tzioumis, A. K. 2005, ApJL, 628, L131

Manchester, R. N., Gaensler, B. M., Wheaton, V. C., et al. 2002, PASA, 19, 207

Morris, T., \& Podsiadlowski, P. 2007, Sci, 315, 1103

Ng, C.-Y., Gaensler, B. M., Murray, S. S., et al. 2009, ApJL, 706, L100

Ng, C.-Y., Gaensler, B. M., Staveley-Smith, L., et al. 2008, ApJ, 684, 481 (N08)

Ng, C.-Y., Potter, T. M., Staveley-Smith, L., et al. 2011a, ApJL, 728, L15

Ng, C.-Y., Potter, T. M., Staveley-Smith, L., et al. 2011b, BAAS, 43, 20.11

Panagia, N. 1999, in IAU Symp. 190, New Views of the Magellanic Clouds, ed. Y.-H. Chu, N. Suntzeff, J. Hesser, \& D. Bohlender (Cambridge: Cambridge Univ. Press), 549

Plait, P. C., Lundqvist, P., Chevalier, R. A., \& Kirshner, R. P. 1995, ApJ, 439, 730

Potter, T. M., Staveley-Smith, L., Ng, C.-Y., et al. 2009, ApJ, 705, 261

Racusin, J. L., Park, S., Zhekov, S., et al. 2009, ApJ, 703, 1752

Sault, R. J., Teuben, P. J., \& Wright, M. C. H. 1995, in ASP Conf. Ser. 77 Astronomical Data Analysis Software and Systems IV, ed. R. A. Shaw, H. E. Payne, \& J. J. E. Hayes (San Francisco, CA: ASP), 433

Staveley-Smith, L., Briggs, D. S., Rowe, A. C. H., et al. 1993a, Natur, 366, 136

Staveley-Smith, L., Gaensler, B. M., Manchester, R. N., et al. 2007, in AIP Conf Proc. 937, Supernova 1987A: 20 Years After: Supernovae and Gamma-Ray Bursters, ed. S. Immler, K. Weiler, \& R. McCray (Melville, NY: AIP), 96

Staveley-Smith, L., Manchester, R. N., Kesteven, M. J., Tzioumis, A. K., \& Reynolds, J. E. R. 1993b, PASAu, 10, 331

Staveley-Smith, L., Manchester, R. N., Kesteven, M. J., et al. 1992, Natur, 355,147

Sugerman, B. E. K., Crotts, A. P. S., Kunkel, W. E., Heathcote, S. R., \& Lawrence, S. S. 2005, ApJS, 159, 60

Suzuki, T., Shigeyama, T., \& Nomoto, K. 1993, A\&A, 274, 883

Tingay, S., Phillips, C., Amy, S., et al. 2009, in 8th International e-VLBI Workshop, Proceedings of Science, 100

Turtle, A. J., Campbell-Wilson, D., Manchester, R. N., Staveley-Smith, L., \& Kesteven, M. J. 1990, IAUC, 5086, 2

Wilson, W. E., Ferris, R. H., Axtens, P., et al. 2011, MNRAS, 416, 832

Zanardo, G., Staveley-Smith, L., Ball, L., et al. 2010, ApJ, 710, 1515

Zanardo, G., Staveley-Smith, L., Ng, C.-Y., et al. 2013, ApJ, 767, 98

Zhekov, S. A., McCray, R., Dewey, D., et al. 2009, ApJ, 692, 1190 